\title{
Materials, Devices, Fabrication, Characterization, and Applications for OLED Illumination and Display
}

\author{
Jwo-Huei Jou, ${ }^{1}$ Yong Qiu, ${ }^{2}$ Saulius Grigalevicius, ${ }^{3}$ and Etienne Baranoff ${ }^{4}$ \\ ${ }^{1}$ Department of Materials Science and Engineering, National Tsing-Hua University, Hsin-Chu 30013, Taiwan \\ ${ }^{2}$ Department of Chemistry, Tsinghua University, Beijing 100084, China \\ ${ }^{3}$ Kaunas University of Technology, Lithuania \\ ${ }^{4}$ École Polytechnique Fédérale de Lausanne, Switzerland \\ Correspondence should be addressed to Jwo-Huei Jou, jjou@mx.nthu.edu.tw
}

Received 19 September 2012; Accepted 19 September 2012

Copyright (C) 2012 Jwo-Huei Jou et al. This is an open access article distributed under the Creative Commons Attribution License, which permits unrestricted use, distribution, and reproduction in any medium, provided the original work is properly cited.

The estimated global revenue is to be $\$ 96$ billion for lighting in 2012, and the same for flat panel display in 2018. Due to the numerous inherently disruptive advantages, such as being a plane light, very high in color rendering index, tunable color temperature, transparent, dimmable, humanfriendly, wide viewing angle, mercury free, sustainable raw materials, ultrathin, light weight, high contrast, fast response time and energy-saving, and so forth, organic light emitting diode (OLED) display and lighting will, sooner or later, take up a major portion of around $\$ 200$ billion revenue and eventually disrupt the current markets. Nowadays, OLED technology is on the threshold of reliability for numerous more contemporary applications. Even OLEDs have already met the requirements for some commercial applications in portable electronics, such as cellular phones, car stereos, digital cameras, and digital watches, there still some challenges especially like further increasing the lifetime and efficiency, while keeping the manufacturing cost lower, quality better, and emissive area size larger.

Recently, phosphorescent OLEDs had received a great deal of attention owing to their potential in achieving high efficiency, such as $100 \%$ internal quantum efficiency or $20 \%$ in external quantum efficiency (EQE), while 5\% EQE for typical fluorescent OLEDs. In their review article, B. Diouf et al. discuss the key factors controlling the efficiency of phosphorescent OLEDs based on green and red emitting iridium complexes. The important factors discussed are exciton confinement, charge trapping, dopant concentration, and molecular structure of the materials. Interestingly, these factors are presented as a full system taking into account the emitter, the host, and the adjacent layers as a whole.

The realization of deep-blue phosphorescent OLEDs has proved challenging. A significant restriction is the broad photoluminance spectrum characteristics of organic dopants. It is important to note that deep-blue or even pure-blue emission has not yet been observed from the phosphorescent emitters containing OLED devices to the moment. As a result, developing high-efficiency deep-blue OLEDs on the basis of phosphorescent dopants becomes crucial. In J. G. Jang and H. J. Ji paper, the authors investigated the deep-blue OLEDs using an emissive layer made of mCP as the host and FCNIr (pic) as the emitter. Optimization of the device architecture was achieved by varying the adjacent hole and electron transport layers, and results in a best device having CIE coordinates $(0.14,0.22)$ and $13.3 \%$ maximum external quantum efficiency.

The fabrication of small molecule based OLEDs generally requires thermal evaporation in a high vacuum. Thermal deposition process enables the formation of well controlled, homogeneous films, and the construction of very complex multilayer structures. This high freedom in layer design, allowing discrete charge transport and charge blocking layers to be formed, is one main reason accounting for the high efficiencies of OLEDs. Owing to the fact that molecular weight is too high for polymers to be thermally evaporated, polymer light emitting diodes (PLEDs) are often fabricated by solution processing techniques, such as spin-coating, inkjet printing, screen printing and rotogravure printing, 
and so forth. Solution processed PLEDs hold significant promises for low cost devices. B. Ch. D. Salert et al. describe the synthesis and optoelectronic properties of a series of novel electron transporting polymers based on triazine moieties grafted on a polystyrene backbone. Furthermore, the polymers have been blended in the emissive layer containing a green emitting iridium complex and $\mathrm{CoH}-001$ as a cohost.

Nowadays, OLED efficacy has already reached the fluorescent tube efficacy, but the lifetime remains a fundamental problem that limits its swiftness in commercialization. Luminance degradation is one of the crucial problems for broad spectrum of OLED lifetime and consistency. In J. Yu et al's paper, the authors investigated blue OLEDs using two dopants in single emitting layer. Higher efficiencies were obtained by codoping the emitting layer when compared to the case of noncodoping. The improvement was attributed to the charge balance in emitting layer. Additionally longer operational lifetime of the devices was achieved due to the expansion of recombination zone in emitting area.

All these research articles represent an innovative, exciting, novel and insightful observation into the state of the art, as well as promising opportunity for contemporary flat panel displays and solid state lightings. As reported in respective papers the performance of OLEDs can easily improve by the use of various efficient organic materials, such as novel dyes, various hosts, electron transporting materials, hole transporting materials, electron injection materials, hole injection materials, or by using a proper device structure and an efficient encapsulation techniques. This special issue is going to contribute significantly for development and commercialization of OLEDs.

Jwo-Huei Jou Yong Qiu Saulius Grigalevicius Etienne Baranoff 

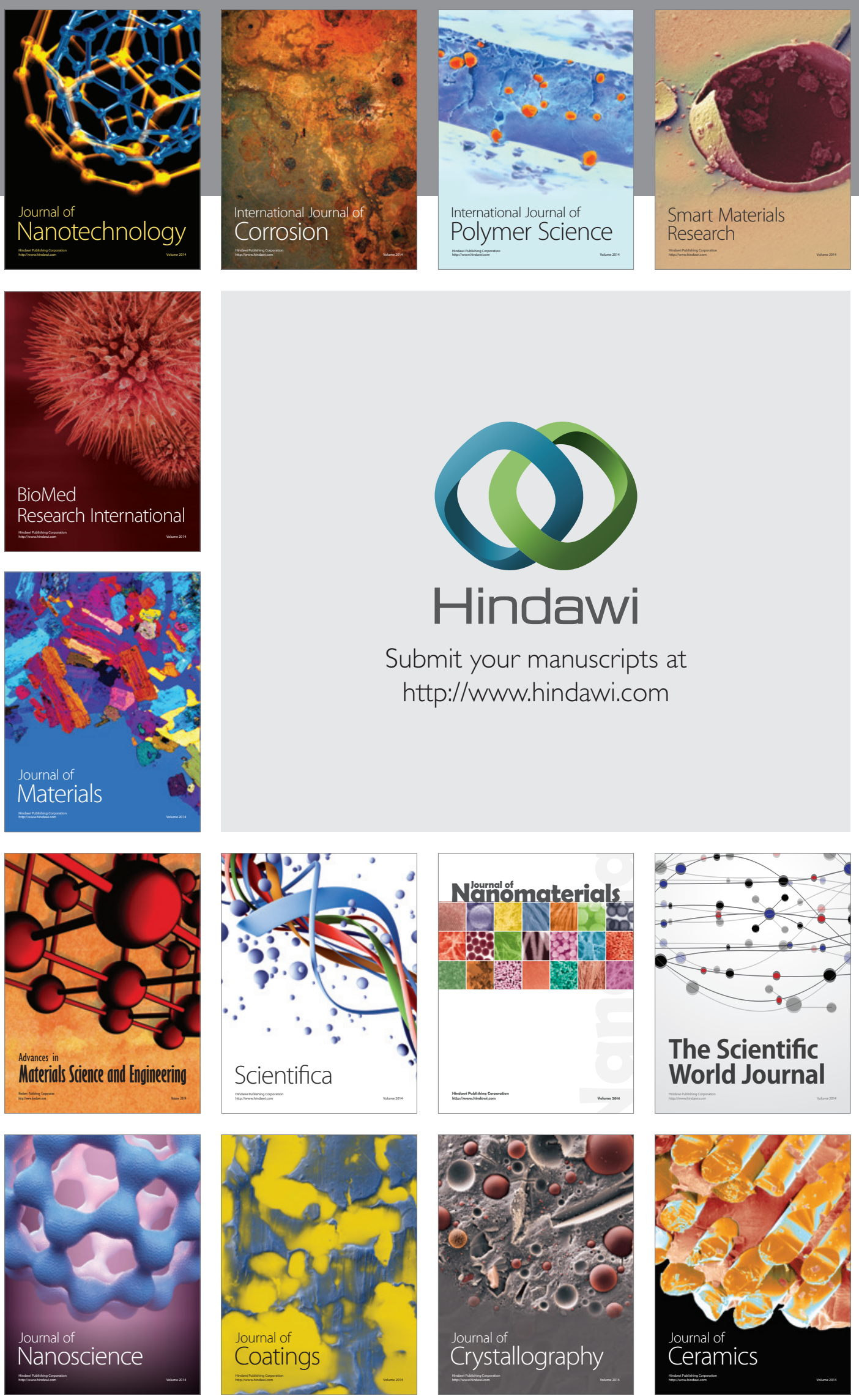

The Scientific World Journal

Submit your manuscripts at

http://www.hindawi.com

\section{World Journal}

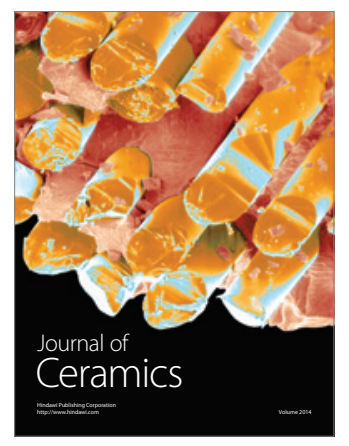

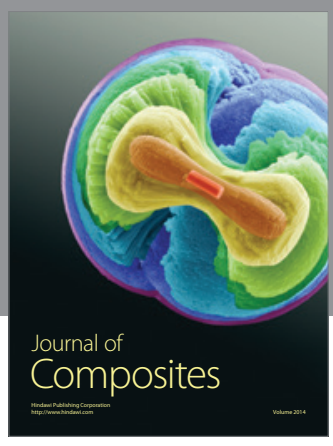
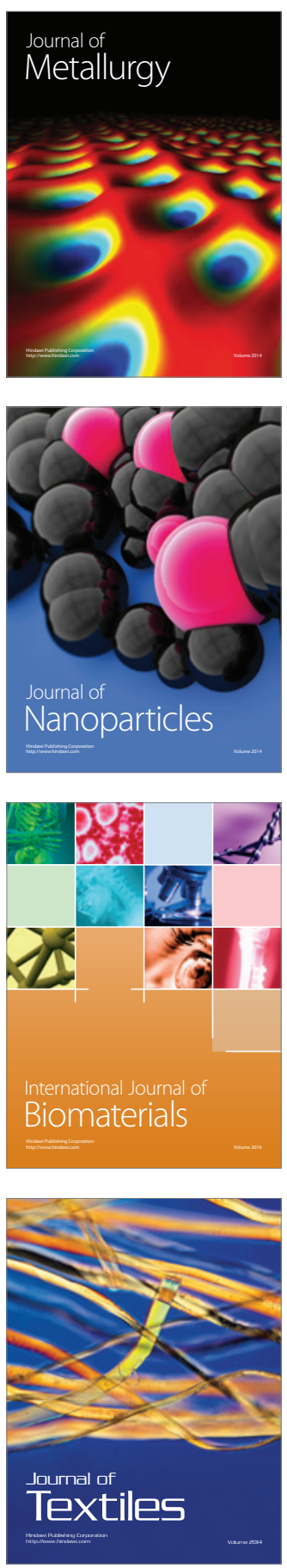